\title{
Abnormal pressure-wave reflection in pregnant women with chronic hypertension: association with maternal and fetal outcomes
}

\author{
Takuji Tomimatsu ${ }^{1}$, Mika Fujime ${ }^{2}$, Tomoko Kanayama ${ }^{2}$, Kazuya Mimura ${ }^{2}$, Shinsuke Koyama ${ }^{2}$, \\ Takeshi Kanagawa ${ }^{2}$, Masayuki Endo ${ }^{2}$, Koichiro Shimoya ${ }^{1}$ and Tadashi Kimura ${ }^{2}$
}

The current study tested the hypothesis that abnormal pressure-wave reflection may have an important role in identifying pregnant women with chronic hypertension who might develop pre-eclampsia (PE) and/or fetal growth restriction. Pulse-wave analyses were performed to assess maternal arterial stiffness during 26-32 weeks of gestation in 41 women with chronic hypertension. We measured the central systolic pressure (CSP) and augmentation index (AIx) noninvasively using pulse waveforms of the radial artery with an automated applanation tonometric system. In a multiple regression analysis that included Alx-75 (Alx at a heart rate of 75 beats per minute), brachial systolic pressure, maternal height, smoking status, gestational age at testing and the presence of antihypertensive treatment at testing as independent determinants, Alx-75 was the only significant determinant of birth weight, whereas the brachial systolic pressure was not. In pregnant women with chronic hypertension who subsequently developed both superimposed PE and fetal growth restriction, CSP, Alx, Alx-75, and the brachial systolic and pulse pressures were all significantly higher than those who did not develop superimposed PE nor small for gestational age. In contrast, Alx-75 was the only significantly elevated hemodynamic parameter in patients who developed fetal growth restriction but not superimposed PE. In addition, CSP was the only significantly elevated hemodynamic parameter in patients who developed superimposed PE but not fetal growth restriction. Abnormal pressure-wave reflection during 26-32 weeks of gestation showed a stronger correlation with birth weight than conventional brachial blood pressure. Our findings might provide new insight into the pathophysiology of fetal growth restriction as well as superimposed PE in pregnancies complicated with chronic hypertension.

Hypertension Research (2014) 37, 989-992; doi:10.1038/hr.2014.109; published online 26 June 2014

Keywords: arterial stiffness; abnormal pressure-wave reflection; chronic hypertension; pregnancy; pulse-wave analysis

\section{INTRODUCTION}

The association between chronic hypertension during pregnancy and increased maternal and neonatal mortality and morbidity is well known. These adverse outcomes are dependent largely on the development of superimposed pre-eclampsia (PE) and/or fetal growth restriction. Although the incidence of superimposed $\mathrm{PE}$ and/or fetal growth restriction apparently increases with the severity of hypertension, mild hypertension does not necessarily indicate a better outcome. $^{1}$

Recently, noninvasive assessment of arterial stiffness by pulse waveform analysis has been increasingly performed in clinical settings. ${ }^{2-4}$ Several reports have established that arterial stiffness, expressed as augmentation index (AIx) and central systolic pressure (CSP), are more strongly and independently correlated with future cardiovascular events than with conventional brachial blood pressure. $^{5-8}$
In pregnant women, we have reported that AIx and CSP showed greater reduction than brachial blood pressure, and AIx is independent of brachial blood pressure throughout pregnancy, suggesting that measurement of AIx might provide distinct information on arterial compliance during pregnancy. ${ }^{9}$ We also found a close association between neonatal birth weight and arterial stiffness during the second trimester in normotensive pregnant women, indicating that abnormal pressure-wave reflection might affect fetal growth even in the absence of hypertension..$^{10}$ In addition, recent studies have reported increased AIx and CSP in women with newly developed PE. ${ }^{11,12}$ Women who develop PE reportedly show abnormal pressure-wave reflection from the first trimester of pregnancy, ${ }^{13,14}$ and those with a history of intrauterine growth restriction and/or early-onset PE show increased AIx up to 6-24 postpartum months. ${ }^{15}$

Based on these findings, we hypothesized that abnormal pressurewave reflection may have an important role in identifying pregnant

${ }^{1}$ Department of Obstetrics and Gynecology, Kawasaki Medical School, Kurashiki, Okayama, Japan and ${ }^{2}$ Department of Obstetrics and Gynecology, Osaka University Graduate School of Medicine, Suita, Osaka, Japan

Correspondence: Dr T Tomimatsu, Department of Obstetrics and Gynecology, Kawasaki Medical School, 577 Matsushima, Kurashiki, Okayama 701-0192, Japan. E-mail: tomimatsu@med.kawasaki-m.ac.jp

Received 25 November 2013; revised 20 February 2014; accepted 8 May 2014; published online 26 June 2014 
women with chronic hypertension who might develop PE and/or fetal growth restriction.

\section{METHODS}

\section{Study participants}

A total of 41 singleton pregnancies presenting at Osaka University Medical Hospital between May 2009 and April 2011 were included in this study. Chronic hypertension was defined as a systolic blood pressure of $\geqslant 140 \mathrm{~mm} \mathrm{Hg}$ and/or a diastolic blood pressure of $\geqslant 90 \mathrm{~mm} \mathrm{Hg}$ before 20 weeks of gestation, documented hypertension before pregnancy, or persistent hypertension after six postpartum weeks. Alpha methyldopa and Ca-channel blockers are the preferred antihypertensive drugs used during pregnancy when therapy was indicated. Diagnosis of superimposed PE required the presence of new-onset proteinuria $(\geqslant 0.3 \mathrm{~g}$ of protein in 24 -h urine collection or a dipstick test result of $\geqslant 1+$, if 24 -h urine collection was not performed) or worsening hypertension in women with proteinuria at baseline. ${ }^{16}$ Neonatal birth weight was expressed as the s.d. of the mean birth weight of Japanese population matched for gestational age, sex and parity. Small for gestational age (SGA) babies were defined as those with a birth weight less than the 10th percentile. ${ }^{17}$ In all the study participants, gestational age was confirmed by ultrasonography before 12 weeks of gestation. Maternal characteristics, including age, parity and gestational age at examination and birth were recorded. All women were followed up until after delivery, and fetal and maternal outcomes were obtained from the women's medical records. All women who participated in this study were of Asian origin. Exclusion criteria were renal disease, thyroid disease, collagen vascular diseases, fetal congenital malformations and chromosomal abnormalities. This study was approved by the Clinical Investigation and Research Ethics Committees of the Faculty of Medicine at Osaka University, and written informed consent was obtained from all subjects.

\section{Pulse-wave analysis}

Pulse-wave analyses were performed during 26-32 weeks of gestation. Arterial pulse waveforms of the right radial artery were measured noninvasively using an automated applanation tonometric system (HEM-9000AI; Omron Healthcare, Kyoto, Japan). With this device, we measured various hemodynamic parameters such as AIx, AIx-75 (AIx at a heart rate of 75 beats per minute) and CSP. The measurement time was approximately $1 \mathrm{~min}$, and the device enabled measurement in the sitting position. The methodology for measuring radial pulse waveforms by this device has been described in detail in previous manuscripts. ${ }^{9} 18-20$ The coefficients of variation of intra- and inter-observer measurements for AIx and CSP using this device were reported to be approximately $2-4 \%,{ }^{18,21}$ indicating good reproducibility. In addition, this device reportedly shows a strong correlation with the AIx values measured with a SphygmoCor system (AtCor Medical, Sydney, NSW, Australia). ${ }^{22}$

Brachial blood pressure was measured simultaneously in the opposite arm with an oscillometric device incorporated in HEM-9000AI. Brachial systolic and diastolic blood pressures were defined by the first and fifth Korotkoff sound, respectively. Brachial pulse pressure, which is considered a surrogate measure of arterial compliance, ${ }^{23}$ was calculated by subtracting the brachial diastolic pressure from the brachial systolic pressure.

\section{Statistical analysis}

Results were expressed as means \pm s.d. and analyzed using one-way analysis of variance, followed by Newman-Keuls post hoc test. Pearson's correlation coefficient $(r)$ was used to determine the correlation between birth weight and CSP, AIx, AIx-75 and the brachial systolic, diastolic and pulse pressures. A multiple regression analysis was performed with birth weight, expressed as s.d. of the mean Japanese birth weight, as the dependent variable and AIx-75, brachial systolic pressure, maternal height, smoking status, gestational age at testing and the presence of antihypertensive treatment at testing as the independent variables to examine which variables independently associated with birth weight. No adjustment for parity was applied in the regression models, because the Japanese growth charts account for parity. Statistical analyses were performed using SPSS 12.0.2 (SPSS, Chicago, IL, USA). A value of $P<0.05$ was considered statistically significant.

\section{RESULTS}

During the study, the prevalence of chronic hypertension in pregnant women was approximately $4 \%$ in our hospital. All women with singleton pregnancies and chronic hypertension in our cohort, pulsewave analyses were successfully performed in the sitting position within 60-90 s.

Table 1 shows the clinical characteristics and maternal and fetal outcomes of the study populations. Maternal age, body mass index at examination, height, parity and gestational age at examination are shown. In our cohort, $27 \%$ of women took some antihypertensive medicine at examinations. Superimposed PE occurred in $16(39 \%)$ patients, and 15 (37\%) babies were SGA. Abruptio placentae occurred in two $(5 \%)$ patients. No eclampsia, fetal demise or HELLP syndrome was noted in our cohort.

In the simple regression analyses between birth weight, expressed as s.d. of the mean Japanese birth weight, and hemodynamic parameters measured during 26-32 weeks of gestation, birth weight was significantly correlated with CSP $(r=-0.41, \quad P=0.009)$, AIx $(r=-0.51, P<0.001)$ and AIx-75 $(r=-0.52, P<0.001)$. However, the correlation was not statistically significant between birth weight and brachial systolic $(r=-0.29, P=0.07)$, diastolic $(r=-0.26$, $P=0.10)$ and pulse pressure $(r=-0.13, P=0.40)$.

To examine the association between birth weight, AIx and brachial blood pressure in more detail, a multiple regression analysis was performed with birth weight, expressed as s.d. of the mean Japanese birth weight, as the dependent variable and AIx-75, brachial systolic pressure, maternal height, smoking status, gestational age at testing and the presence of antihypertensive treatment at testing as the independent variables to examine which variables independently associated with neonatal birth weight. As can be seen in Table 2, AIx-75 was the only significant determinant of neonatal birth weight among the indicated variables, whereas the brachial systolic pressure was not.

Then we stratified the study cohort into four groups according to the development of superimposed PE and/or SGA status, that is, PE with SGA group, PE without SGA group, SGA without PE group and control (neither PE nor SGA) group. Table 3 compares the hemodynamic parameters of women with chronic hypertension who did or did not develop superimposed PE and/or SGA, measured during 2632 weeks of gestation. In pregnant women with chronic hypertension who subsequently developed both superimposed PE and SGA (PE

\section{Table 1 Characteristics of all study populations}

\begin{tabular}{lc}
\hline Clinical characteristics at examination & \\
Age (years) & $36.3 \pm 4.1$ \\
BMI at testing $\left(\mathrm{kg} \mathrm{m}^{-2}\right.$ ) & $27.8 \pm 6.2$ \\
Height (meters) & $1.58 \pm 0.05$ \\
Nulliparity (\%) & $37 \%$ \\
Gestational age at testing (week) & $29.4 \pm 2.2$ \\
Any antihypertensive therapies at testing, $n$ (\%) & $11(27 \%)$ \\
& \\
Maternal and fetal outcomes & \\
Development of superimposed pre-eclampsia, $n(\%)$ & $16(39 \%)$ \\
Development of abruptio placentae (\%) & $2(5 \%)$ \\
Gestational age at birth (week) & $37.1 \pm 2.7$ \\
Birth weight (g) & $2402 \pm 708$ \\
SGA babies, $n$ (\%) & $15(37 \%)$ \\
s.d. of birth weight & $-0.83 \pm 0.95$
\end{tabular}

Abbreviations: BMI, body mass index; SGA, small for gestational age. Data are presented as mean \pm s.d. or $\%$. 
Table 2 Multiple regression analysis with s.d. of the mean Japanese birth weight as the dependent variable

\begin{tabular}{lrrrr}
\hline & & \multicolumn{3}{c}{ Standard } \\
& Coefficient & s.e. & coefficient & P \\
\hline Intercept & -3.534 & 5.89 & & 0.55 \\
Alx-75 & -0.380 & 0.01 & -0.45 & 0.01 \\
Maternal height & 0.029 & 0.03 & 0.15 & 0.38 \\
Smoking & 0.282 & 0.44 & 0.10 & 0.53 \\
Gestational age at testing & 0.100 & 0.07 & 0.23 & 0.15 \\
Any antihypertensive therapies at testing & 0.211 & 0.32 & 0.10 & 0.52 \\
Brachial systolic pressure & -0.016 & 0.01 & -0.26 & 0.12
\end{tabular}

Abbreviation: Alx-75, Alx at a heart rate of 75 beats per minute.

Table 3 Hemodynamic parameters of women with chronic hypertension who did or did not develop PE and/or SGA, measured during 26-32 weeks' gestation

\begin{tabular}{lcccc}
\hline & $\begin{array}{c}\text { PE with SGA } \\
(\mathrm{n}=9)\end{array}$ & $\begin{array}{c}\text { PE without } \\
\text { SGA }(\mathrm{n}=7)\end{array}$ & $\begin{array}{c}\text { SGA without } \\
\text { PE }(\mathrm{n}=6)\end{array}$ & \multicolumn{1}{c}{$\begin{array}{c}\text { Control } \\
(\mathrm{n}=19)\end{array}$} \\
\hline $\mathrm{CSP}(\mathrm{mm} \mathrm{Hg})$ & $151.2 \pm 12.4^{\mathrm{a}}$ & $141.6 \pm 21.2^{\mathrm{a}}$ & $137.3 \pm 11.6$ & $124.8 \pm 14.9$ \\
Alx (\%) & $78.1 \pm 8.4^{\mathrm{a}}$ & $71.1 \pm 3.3$ & $76.3 \pm 14.6$ & $65.4 \pm 13.1$ \\
$\begin{array}{l}\text { Alx-75 (\%) } \\
\text { Brachial systolic } \\
\text { pressure (mm Hg) }\end{array}$ & $147.4 \pm 11.8^{\mathrm{a}}$ & $138.7 \pm 22.3$ & $133.5 \pm 8.5$ & $126.3 \pm 12.5$ \\
$\begin{array}{l}\text { Brachial diastolic } \\
\text { pressure (mm Hg) }\end{array}$ & $94.0 \pm 12.0$ & $94.9 \pm 16.7$ & $91.3 \pm 8.2$ & $81.9 \pm 11.2$ \\
$\begin{array}{l}\text { Brachial pulse pres- } \\
\text { sure (mm Hg) }\end{array}$ & $53.4 \pm 8.7^{\mathrm{a}}$ & $43.9 \pm 10.8$ & $42.2 \pm 6.1$ & $44.4 \pm 6.4$ \\
\hline
\end{tabular}

Abbreviations: Alx, augmentation index; Alx-75, Alx at heart rate of 75 beats per minute; CSP, central systolic pressure; PE, pre-eclampsia; SGA, Small for gestational age.

Data are presented as mean \pm s.d..

Significant difference from control $(P<0.05)$

with SGA group), CSP, AIx, AIx-75 and the brachial systolic and pulse pressures were all significantly higher than those in the control group, and CSP, AIx, and the brachial systolic and pulse pressures showed the highest values among the groups. In contrast, AIx-75 was the only significantly elevated hemodynamic parameter in patients who developed SGA but not superimposed PE (SGA without PE group). In addition, CSP was the only significantly elevated hemodynamic parameter in patients who developed superimposed PE but not SGA (PE without SGA group).

\section{DISCUSSION}

As mentioned above, adverse maternal and fetal outcomes in pregnancies complicated with chronic hypertension have been associated with the occurrence of superimposed PE and/or fetal growth restriction. Therefore, identification of pregnant women with an increased risk of these complications would result in more intensive monitoring and early recognition before severe complications develop. In this study, we have shown that abnormal pressure-wave reflection might be involved in the pathogenesis of superimposed PE and/or fetal growth restriction, and that abnormal pressure-wave reflection has a role in identifying pregnant women who might develop these complications. In addition, abnormal wave reflection is the only independent predictor of low birth weight in pregnant women with chronic hypertension when measured at 26-32 weeks of gestation. Increased maternal peripheral resistance has been shown using impedance cardiography in pregnancies complicated by severe fetal growth restriction and/or PE. ${ }^{24}$ Our findings might also provide a new insight into the pathophysiology of fetal growth restriction as well as superimposed PE in pregnant women with chronic hypertension.

Brachial blood pressure measurement has long been an integral part of antenatal care, as the diagnosis of both $\mathrm{PE}$ and chronic hypertension is primarily based on the brachial blood pressure. In addition, several studies suggest that adverse perinatal and maternal outcomes in pregnancies complicated with chronic hypertension are related to the severity of hypertension, that is, elevated brachial blood pressure. ${ }^{25}$ On the other hand, it is widely known that elevated brachial blood pressure is not necessarily related to serious adverse maternal and fetal outcomes such as eclampsia, abruptio placentae, fetal demise and HELLP syndrome, and that a significant proportion of women with PE can carry a pregnancy nearly to term without complications. ${ }^{26}$ Therefore, additional markers to establish or rule out imminent adverse outcomes, which are more important than making a diagnosis of PE or chronic hypertension, are needed for timely intervention.

Recently, generalized endothelial dysfunction caused by excess antiangiogenic and reduced pro-angiogenic factors from hypoxic placenta has been shown to play a central role in the pathogenesis of PE. ${ }^{27-29}$ Furthermore, the severity of imbalance of these circulating angiogenic factors assessed by the ratio of soluble fms-like tyrosine kinase and placental growth factor, has been reported to predict adverse outcomes better than the severity of elevated brachial blood pressure. $^{30}$ Even in normotensive pregnant women, endothelial dysfunction has been shown to impair fetal growth, ${ }^{31}$ and the levels of anti-angiogenic factors in maternal serum have been reported to correlate with neonatal birth weight. ${ }^{32}$

Arterial stiffness (expressed as CSP and AIx) is determined by the magnitude and timing of the reflected pulse waveform of both the conduit and resistance arteries, and is thought to represent endothelial function of the whole systemic arterial tree. ${ }^{33,34}$ It has been reported that endothelial dysfunction is the first step toward atherosclerosis in patients with chronic hypertension. ${ }^{35}$ In the present study, abnormal pressure-wave reflection was the only significant determinant of birth weight. Based on the present study, abnormal pressure-wave reflection, which may reflect endothelial dysfunction and relatively early changes associated with atherosclerosis, might predict fetal outcome better than brachial blood pressure in pregnant women with chronic hypertension. Even in predicting the development of superimposed PE, which is diagnosed mainly by the brachial blood pressure, CSP was the only significantly elevated hemodynamic parameter.

Severe chronic hypertension must be treated even during pregnancy, although there is minimal evidence that maternal or neonatal outcomes are improved by simply lowering brachial blood pressure and that the incidence of superimposed PE is affected by antihypertensive drugs. ${ }^{1,25}$ Alpha methyldopa, a central-acting adrenergicblocking agent, is frequently used to control severe chronic hypertension in pregnancy because it is well known for its safe maternal and fetal use. Recently, antihypertensive therapy with alpha methyldopa was shown to not only maintain uteroplacental circulation, ${ }^{36}$ but also improve arterial stiffness in women with PE. ${ }^{12}$ On the other hand, the use of atenolol, a $\beta$-blocker has been reportedly associated with a higher incidence of growth-restricted neonates, ${ }^{37}$ and is not recommended for pregnant women with chronic hypertension. ${ }^{38}$ Recently, the large-scale Anglo-Scandinavian Cardiac Outcomes Trial-Conduit Artery Function Evaluation study demonstrated that despite virtually identical reductions in brachial blood pressure, those randomized to an atenolol-based regimen had a higher CSP than those randomized to an amlodipine-based regimen, 
which might have contributed to the significantly increased total and cardiovascular mortality in the atenolol group. ${ }^{5}$ These and the present findings suggest that atenolol may be less effective for lowering arterial stiffness, which may have an unfavorable effect on fetal growth in patients with chronic hypertension.

In summary, we have demonstrated that abnormal pressure-wave reflection assessed by pulse-wave analyses during 26-32 weeks of gestation showed a stronger correlation with neonatal birth weight than with conventional brachial blood pressure in patients with chronic hypertension. Maternal cardiovascular impairment such as a low output and high resistance circulatory state have been reported in pregnancy complicated by severe fetal growth restriction. ${ }^{39}$ Although the association between these maternal cardiovascular characteristics and the abnormal wave reflection observed in the present study remains unclear, these cardiovascular changes might be important in terms of the relative increased long-term cardiovascular risk in women with a history of fetal growth restriction. ${ }^{40}$ A larger study combining pulse-wave analysis with the assessment of angiogenic imbalance, which is assumed to have a close association to endothelial dysfunction, might prove our hypothesis that the pathogenesisoriented parameters might better predict adverse maternal and fetal outcomes in pregnancies complicated with chronic hypertension.

\section{ACKNOWLEDGEMENTS}

This study was supported in part by Grant-in-Aid for General Scientific Research (T23592402, T25462555, T25462556, T24592493 T25462556 and A00249080) from the Ministry of Education, Culture, Sports, Science and Technology of Japan.

1 Cunningham FG, Lenko KJ, Bloom SH, Hauth JC, Gilstrap L III and Wenstrom KD (eds). Chronic Hypertension. In: Williams obstetrics (23rd edn) McGraw-Hill, New York 2010, pp 983-995.

2 Kelly R, Hayward CS, Avolio A, O'Rourke MF. Non-invasive registration of the arterial pressure pulse waveform using high-fidelity applanation tonometry. J Vasc Med Biol 1989; 1: 142-149.

3 Nichols WW. Clinical measurement of arterial stiffness obtained from noninvasive pressure waveforms. Am J Hypertens 2005; 18: 3S-10S.

4 Tomiyama H, Yamashina A. Non-invasive vascular function tests: their pathophysiological background and clinical application. Circ J 2010; 74: 24-33.

5 Williams B, Lacy PS, Thom SM, Cruickshank K, Stanton A, Collier D, Hughes AD, Thurston H, O'Rourke M. Differential impact of blood pressure-lowering drugs on central aortic pressure and clinical outcomes: principal results of the Conduit Artery Function Evaluation (CAFE) study. Circulation 2006; 113: 1213-1225.

6 Roman MJ, Devereux RB, Kizer JR, Lee ET, Galloway JM, Ali T, Umans JG, Howard BV Central pressure more strongly relates to vascular disease and outcome than does brachial pressure: the Strong Heart Study. Hypertension 2007; 50: 197-203.

7 Chirinos JA, Zambrano JP, Chakko S, Veerani A, Schob A, Willens HJ, Perez G, Mendez AJ. Aortic pressure augmentation predicts adverse cardiovascular events in patients with established coronary artery disease. Hypertension 2005; 45: 980-985.

8 London GM, Blacher J, Pannier B, Guérin AP, Marchais SJ, Safar ME. Arterial wave reflections and survival in end-stage renal failure. Hypertension 2001; 38: 434-438.

9 Fujime M, Tomimatsu T, Okaue Y, Koyamal S, Kanagawa T, Taniguchi T, Kimura T. Central aortic blood pressure and Augmentation index during normal pregnancy. Hypertens Res 2012; 35: 633-638.

10 Tomimatsu T, Fujime M, Kanayama T, Mimura K, Koyama S, Kanagawa T, Kimura T. Maternal arterial stiffness in normotensive pregnant women who subsequently deliver babies that are small for gestational age. Eur J Obstet Gynecol Reprod Biol 2013; 169 24-27.

11 Spasojevic M, Smith SA, Morris JM, Gallery ED. Peripheral arterial pulse wave analysis in women with pre-eclampsia and gestational hypertension. BJOG 2005; 112: 1475-1478.

12 Khalil A, Jauniaux E, Harrington K. Antihypertensive therapy and central hemodynamics in women with hypertensive disorders in pregnancy. Obstet Gynecol 2009, 113: 646-654

13 Khalil AA, Cooper DJ, Harrington KF. Pulse wave analysis: a preliminary study of novel technique for the prediction of pre-eclampsia. BJOG 2009; 116: 268-276.

14 Khalil A, Akolekar R, Syngelaki A, Elkhouli M, Nicolaides KH. Maternal hemodynamics at 11-13 weeks' gestation and risk of pre-eclampsia. Ultrasound Obstet Gynecol 2012 40: 28-34.
15 Yinon Y, Kingdom JC, Odutayo A, Moineddin R, Drewlo S, Lai V, Cherney DZ, Hladunewich MA. Vascular dysfunction in women with a history of preeclampsia and intrauterine growth restriction: insights into future vascular risk. Circulation 2010; 122: 1846-1853.

16 Brown MA, Lindheimer MD, de Swiet M, Van Assche A, Moutquin JM. The classification and diagnosis of the hypertensive disorders of pregnancy: statement from the International Society for the Study of Hypertension in Pregnancy (ISSHP). Hypertens Pregnancy 2001; 20: IX-XIV.

17 Nishida H, Sakanoue M, Kurachi K, Asada A, Kudo S, Funakawa H. Fetal growth curve of Japanese. Nippon Shinseiji Gakkai Zasshi 1984; 20: 90-97.

18 Funada J, Takata Y, Hashida H, Matsumoto Y, Sato S, Hiasa G, Inoue K, Higaki J, Okayama $\mathrm{H}$. Dysfunctional central hemodynamic regulation after daily meal intake in metabolic syndrome. Atherosclerosis 2010; 210: 268-273.

19 Takazawa K, Kobayashi H, Shindo N, Tanaka N, Yamashina A. Relationship between radial and central arterial pulse wave and evaluation of central aortic pressure using the radial arterial pulse wave. Hypertens Res 2007; 30: 219-228.

20 Kohara K, Tabara Y, Oshiumi A, Miyawaki Y, Kobayashi T, Miki T. Radial augmentation index: a useful and easily obtainable parameter for vascular aging. Am J Hypertens 2005; 18: 11S-14S.

21 Sugawara J, Komine H, Hayashi K, Maeda S, Matsuda M. Relationship between augmentation index obtained from carotid and radial artery pressure waveforms. J Hypertens 2007; 25: 375-381.

22 Richardson CJ, Maki-Petaja KM, McDonnell BJ, Hickson SS, Wilkinson IB, McEniery CM. Comparison of estimates of central systolic blood pressure and periphera augmentation index obtained from the Omron HEM-9000AI and SphygmoCor systems. Artery Res 2009; 3: 24-31.

23 Domanski MJ, Davis BR, Pfeffer MA, Kastantin M, Mitchell GF. Isolated systolic hypertension: prognostic information provided by pulse pressure. Hypertension 1999; 34: 375-380

24 Jia RZ, Liu XM, Wang X, Wu HQ. Relationship between cardiovascular function and fetal growth restriction in women with pre-eclampsia. Int J Gynaecol Obstet 2010; 110: 61-63.

25 August $\mathrm{P}$, Lindheimer MD. Chronic hypertension in pregnancy. In Lindheimer MD, Roberts JM and Cunningham FG (eds) Chasley's Hypertensive Disorders in Pregnancy (2nd edn) Appleton \& Lange, Stamford, CT, 1999, p 605.

26 Sibai BM, Stella CL. Diagnosis and management of atypical preeclampsia-eclampsia. Am J Obstet Gynecol 2009; 200: e1-e7.

27 Maynard SE, Min JY, Merchan J, Lim KH, Li J, Mondal S, Libermann TA, Morgan JP, Sellke FW, Stillman IE, Epstein FH, Sukhatme VP, Karumanchi SA. Excess placenta soluble fms-like tyrosine kinase 1 (sFlt1) may contribute to endothelial dysfunction, hypertension, and proteinuria in preeclampsia. J Clin Invest 2003; 111: 649-658.

28 Levine RJ, Lam C, Qian C, Yu KF, Maynard SE, Sachs BP, Sibai BM, Epstein FH, Romero R, Thadhani R, Karumanchi SACPEP Study Group. Soluble endoglin and other circulating antiangiogenic factors in preeclampsia. N Engl J Med 2006; 355 992-1005.

29 Venkatesha S, Toporsian M, Lam C, Hanai J, Mammoto T, Kim YM, Bdolah Y, Lim KH, Yuan HT, Libermann TA, Stillman IE, Roberts D, D'Amore PA, Epstein FH, Sellke FW, Romero R, Sukhatme VP, Letarte M, Karumanchi SA. Soluble endoglin contributes to the pathogenesis of preeclampsia. Nat Med 2006; 12: 642-649.

30 Rana S, Powe CE, Salahuddin S, Verlohren S, Perschel FH, Levine RJ, Lim KH, Wenger JB, Thadhani R, Karumanchi SA. Angiogenic factors and the risk of adverse outcomes in women with suspected preeclampsia. Circulation 2012; 125: 911-919.

31 Koopmans CM, Blaauw J, van Pampus MG, Rakhorst G, Aarnoudse JG. Abnorma endothelium-dependent microvascular dilator reactivity in pregnancies complicated by normotensive intrauterine growth restriction. Am J Obstet Gynecol 2009; 200: e1-e6.

32 Stepan H, Krämer T, Faber R. Maternal plasma concentrations of soluble endoglin in pregnancies with intrauterine growth restriction. J Clin Endocrinol Metab 2007; 92: 2831-2834.

33 McEniery CM, Wallace S, Mackenzie IS, McDonnell B, Yasmin, Newby DE, Cockcroft JR, Wilkinson IB. Endothelial function is associated with pulse pressure, pulse wave velocity, and augmentation index in healthy humans. Hypertension 2006; 48: 602-608.

34 Soga J, Nakamura S, Nishioka K, Umemura T, Jitsuiki D, Hidaka T, Teragawa H, Takemoto H, Goto C, Yoshizumi M, Chayama K, Higashi Y. Relationship between augmentation index and flow-mediated vasodilation in the brachial artery. Hypertens Res 2008; 31: 1293-1298.

35 Davignon J, Ganz P. Role of endothelial dysfunction in atherosclerosis. Circulation 2004; 109: III27-III32.

36 Khalil A, Harrington K, Muttukrishna S, Jauniaux E. Effect of antihypertensive therapy with alpha-methyldopa on uterine artery Doppler in pregnancies with hypertensive disorders. Ultrasound Obstet Gynecol 2010; 35: 688-694.

37 Lydakis C, Lip GY, Beevers M, Beevers DG. Atenolol and fetal growth in pregnancies complicated by hypertension. Am J Hypertens 1999; 12: 541-547.

38 American College of Obstetrics and Gynecologists. Chronic hypertension in pregnancy. Practice Bulletin No.29. Obstet Gynecol 2001; 98: 177-185.

39 Melchiorre K, Sutherland GR, Liberati M, Thilaganathan B. Maternal cardiovascular impairment in pregnancies complicated by severe fetal growth restriction. Hypertension 2012; 60: 437-443

40 Smith GC, Pell JP, Walsh D. Pregnancy complications and maternal risk of ischaemic heart disease: a retrospective cohort study of 129,290 births. Lancet 2001; 357: 2002-2006. 